\title{
Oxaprozin Potassium
}

National Cancer Institute

\section{Source}

National Cancer Institute. Oxaprozin Potassium. NCI Thesaurus. Code C48008.

The potassium salt form of oxaprozin and a member of the propionic acid group of nonsteroidal anti-inflammatory drugs (NSAIDs) with anti-inflammatory, analgesic and antipyretic properties. Oxaprozin potassium reversibly and competitively inhibits cyclooxygenases (COX), thereby blocking the conversion of arachidonic acid to proinflammatory prostag landins. This inhibits the formation of prostaglandins that are involved in pain, inflammation and fever. 